\title{
PELABELAN SUPER MEAN PADA GENERALISASI GRAF TUNAS KELAPA
}

\author{
D.A. Merdekawati ${ }^{1}$, I.W. Sudarsana ${ }^{2}$, dan S. Musdalifah ${ }^{3}$ \\ 1,2,3 Program Studi Matematika Jurusan Matematika FMIPA Universitas Tadulako \\ Jalan Soekarno-Hatta Km. 09 Tondo, Palu 94118, Indonesia. \\ 1'deanmbakyuu@gmail.com, 22sudarsanaiwayan@yahoo.co.id, 35elvymusdalifah@yahoo.com
}

\section{ABSTRACT}

Let $G$ be $a(p, q)$ graf and let $f: V(G) \rightarrow\{1,2, \ldots, n\}$ be an injection. For each edge $e=u v$, let $f^{*}(e)=\frac{f(u)+f(v)}{2}$ if $f(u)+f(v)$ is even, and $f^{*}(e)=\frac{f(u)+f(v)+1}{2}$ if $f(u)+f(v)$ is odd. Then $f$ is called a Super Mean if $f(V) \cup\left\{f^{*}(e): e \in\right.$ $E(G)\}=\{1,2,3, \ldots, p+q\}$. Further investigation will be conducted Super Mean labeling of the Combined the Generalizing Coconut Sprout Graph denoted as $\mathrm{sGCR}_{\mathrm{n}, \mathrm{m}, \mathrm{t}} ; \mathrm{GCR}_{\mathrm{n}, \mathrm{m}, \mathrm{a}, 2}$ and $\mathrm{GCR}_{\mathrm{n}, \mathrm{m}, \mathrm{a}, 1} \cup \mathrm{P}_{\mathrm{u}}$. Graph having Super Mean labeling is called Super Mean graph. In this paper, we showed that the total graph of $\mathrm{sGCR}_{n, m, t}$ for $n \geq$ $5, \mathrm{~m} \geq 2, \mathrm{t}=\left\lfloor\frac{\mathrm{m}}{2}\right\rfloor$ and $\mathrm{s} \geq 1 ; \mathrm{GCR}_{\mathrm{n}, \mathrm{m}, \mathrm{a}, 2}$ for $\mathrm{n} \geq 7$ odd, $\mathrm{m}=\mathrm{n}-3$ and $\mathrm{a}=2 ; \mathrm{GCR}_{\mathrm{n}, \mathrm{m}, \mathrm{a}, 2}$ for $\mathrm{n} \geq 9$ odd, $\mathrm{m}=\mathrm{n}-6$ and $\mathrm{a}=3 ; \mathrm{GCR}_{\mathrm{n}, \mathrm{m}, \mathrm{a}, 2}$ for $\mathrm{n} \geq 9$ odd, $\mathrm{m}=\mathrm{n}-7$ and $\mathrm{a}=4 ; \mathrm{GCR}_{\mathrm{n}, \mathrm{m}, \mathrm{a}, 1} \cup \mathrm{P}_{\mathrm{u}}$ for $\mathrm{n} \geq 9, \mathrm{~m}=6, \mathrm{a}=\mathrm{n}-6, \mathrm{u}=\frac{\mathrm{n}-5}{2}$ is Super Mean.

Keywords $\quad$ : Coconut Sprout Generalized Graph, $\mathbf{s G C R}_{\mathbf{n}, \mathbf{m}, \mathrm{t}}$ Graph, $\mathbf{G C R}_{\mathbf{n}, \mathbf{m}, \mathrm{a}, \mathrm{t}}$ Graph, Super Mean

\section{ABSTRAK}

Misalkan $G$ adalah $(p, q)$ graf dan $f: V(G) \rightarrow\{1,2, \ldots, n\}$ sebuah fungsi injektif. Untuk setiap sisi $e=u v$, maka $\frac{f(u)+f(v)}{2}$ jika $f(u)+f(v)$ genap, dan $\frac{f(u)+f(v)+1}{2}$ jika $f(u)+f(v)$ ganjil. Maka $f$ disebut pelabelan Super Mean jika $f(V) \cup\left\{f^{*}(e): e \in E(G)\right\}=\{1,2,3, \ldots, p+q\}$. Selanjutnya akan dilakukan investigasi pelabelan Super Mean pada gabungan Generalisasi Graf Tunas Kelapa yang dinotasikan dengan $s G C R_{n, m, t} ; G C R_{n, m, a, 2}$ dan $G C R_{n, m, a, 1} \cup P_{u}$. Graf yang mempunyai pelabelan Super Mean disebut graf Super Mean. Pada penelitian ini telah berhasil ditunjukan bahwa graf $s G C R_{n, m, t}$ untuk $n \geq 5, m \geq 2, t=\left\lfloor\frac{m}{2}\right\rfloor$ dan $s \geq 1 ; G C R_{n, m, a, 2}$ untuk $n \geq 7$ ganjil, $m=n-3$ dan $a=2 ; G C R_{n, m, a, 2}$ untuk $n \geq 9$ ganjil, $m=n-6$ dan $a=3 ; G C R_{n, m, a, 2}$ untuk $n \geq 9$ ganjil, $m=n-7$ dan $a=4$; $G C R_{n, m, a, 1} \cup P_{u}$ untuk $n \geq 9, m=6, a=n-6, u=\frac{n-5}{2}$ adalah Super Mean.

Kata Kunci $\quad$ : Generalisasi graf Tunas Kelapa, Graf $s G C R_{n, m, t}$, Graf $G C R_{n, m, a, t}$, Super Mean

\section{PENDAHULUAN}

Secara umum, Graf adalah suatu diagram yang memuat informasi tertentu jika diinterpretasikan secara tepat. Dalam kehidupan sehari-hari, graf digunakan untuk menggambarkan 
berbagai macam struktur yang ada. Tujuannya adalah sebagai visualisasi objek-objek agar dapat lebih mudah dipahami.

Dalam pengembangannya dikenal pula pelabelan graf yang kini menjadi topik yang mendapat banyak perhatian. Model-model yang terdapat pada pelabelan graf diaplikasikan dalam cakupan yang luas seperti dalam masalah peta jaringan jalan raya, jaringan internet, sistem alamat jaringan komunikasi, kriptografi, dan lain sebagainya.

Pelabelan graf pertama kali diperkenalkan oleh Sadlàčk (1964), kemudian Stewart (1966), Kotzig dan Rosa (1970). Pelabelan merupakan pemetaan bijeksi yang memetakan unsur himpunan titik dan atau unsur himpunan sisi ke bilangan asli yang disebut label. Pelabelan menurut domainnya dibagi menjadi tiga yaitu pelabelan titik, pelabelan sisi, dan pelabelan total. Pelabelan titik adalah pelabelan dengan domain himpunan titik, pelabelan sisi adalah pelabelan dengan domain himpunan sisi, dan pelabelan total adalah pelabelan dengan domain gabungan himpunan titik dan himpunan sisi (Wallis et al, 2000)

Berbagai macam hasil pengembangan dan kajian dari metode pelabelan graf antara lain: Pelabelan Total Sisi Ajaib Super (TSAS), Pelabelan Total Titik Ajaib, Pelabelan Total Sisi Anti Ajaib Super (TSAAS), Pelabelan Graceful, Pelabelan Harmoni, Pelabelan Prime Cordial, Pelabelan Geometric Mean, Pelabelan Super Harmonic Mean, dan Pelabelan Super Mean.

Pelabelan mean pertama kali diperkenalkan secara umum oleh Somasundaram dan Ponraj (2003). Sementara pelabelan Super Mean pertama kali diperkenalkan oleh Ramya et al (2012). Misalkan $G$ adalah $(p, q)$ graf dan $f: V(G) \rightarrow\{1,2, \ldots, n\}$ sebuah fungsi injektif. Untuk setiap sisi $e=$ $u v$, maka

$f^{*}(e)= \begin{cases}\frac{f(u)+f(v)}{2} & \text { jika } \mathrm{f}(\mathrm{u})+\mathrm{f}(\mathrm{v}) \text { genap } \\ \frac{f(u)+f(v)+1}{2} & \text { jika } \mathrm{f}(\mathrm{u})+\mathrm{f}(\mathrm{v}) \text { ganjil }\end{cases}$

Selanjutnya $f$ disebut pelabelan Super Mean jika $f(V) \cup\left\{f^{*}(e): e \in E(G)\right\}=\{1,2,3, \ldots, p+q\}$. (Ramya dan Jeyanthi, 2012).

Isnawati, dkk mengeluarkan konjektur mengenai graf Tunas Kelapa Tunggal dengan pelabelan total super (a,d) sisi anti-magic (Lestari, 2013). Oleh karena itu penulis tertarik melakukan kajian Pelabelan Super Mean pada Generalisasi Graf Tunas Kelapa. 


\section{METODE PENELITIAN}

1. Memulai penelitian, berupa studi literatur

2. Mengembangkan bentuk baru dari graf Tunas Kelapa yang disebut generalisasi graf Tunas Kelapa

3. Menotasikan titik dan sisi pada generalisasi graf Tunas Kelapa

4. Menemukan pola pelabelan Super Mean pada generalisasi graf Tunas Kelapa

5. Membuat fungsi pelabelan yang dilengkapi dengan bukti

6. Memberikan kesimpulan hasil penelitian

7. Selesai

\section{HASIL DAN PEMBAHASAN}

Pada bagian ini akan dibahas mengenai pelabelan Super Mean generalisasi graf Tunas Kelapa yang dinotasikan dengan $G C R_{n, m, a, t}$ dimana $V\left(G C R_{n, m, a, t}\right)=\left\{x_{i}, y_{j}, z_{k}^{l} ; 1 \leq i \leq n, 1 \leq j \leq\right.$ $m, 1 \leq k \leq t, 1 \leq l \leq a\} \quad$ dan $E\left(G C R_{n, m, a, t}\right)=\left\{x_{n} x_{i,} ;=1\right\} \cup\left\{x_{i} x_{i+1} ; 1 \leq i \leq n-1\right\} \cup\left\{x_{n} y_{j} ; 1 \leq j \leq\right.$ $m\} \cup\left\{y_{j} y_{j+1} ; 1 \leq j \leq m-1\right\} \cup\left\{z_{k}^{l} z_{k+1}^{l} ; 1 \leq k \leq t-1 ; 1 \leq l \leq a\right\} \cup\left\{x_{n} z_{k}^{l} ; 1 \leq l \leq a\right\}$.

\subsection{Penotasian Titik dan Sisi pada Graf $G_{C} R_{n, m, a, t}$}

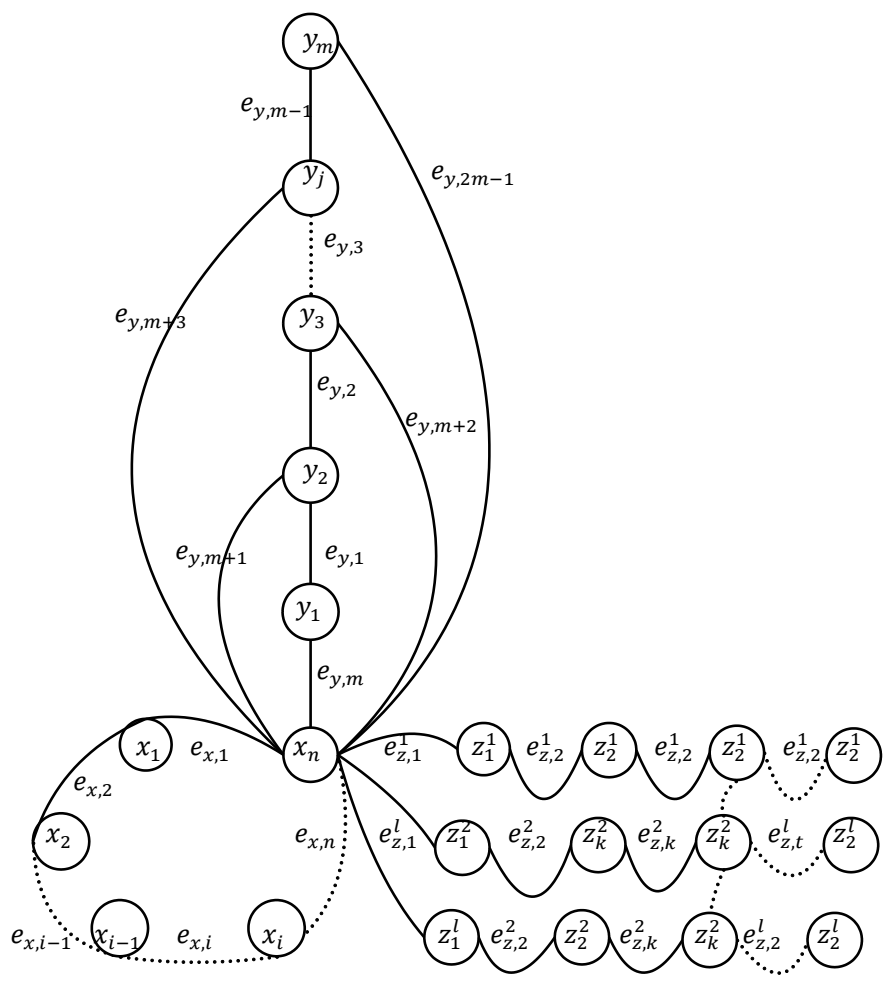

Gambar 1 : Penotasian Generalisasi graf Tunas Kelapa $G C R_{n, m, a, t}$ 
3.1.1. Pelabelan Super Mean Pada Graf $s G C R_{n, m, t}$

Teorema 1 : Graf $s G C R_{n, m, t}$ untuk $n \geq 5, m \geq 2, t=\left\lfloor\frac{m}{2}\right\rfloor$ dan $s \geq 1$ adalah Super mean.

\section{Bukti :}

Berdasarkan penotasian titik dan sisi graf $s G C R_{n, m, t}$ bentuk fungsi injektif $f\left(V\left(s G C R_{n, m, t}\right)\right) \cup\left\{f^{*}(e): e \in E\left(s G C R_{n, m, t}\right)\right\} \rightarrow\{1,2,3, \ldots, s(2 n+3 m+2 t-1)-$ $m+2 t\}$ sebagai berikut :

$$
f_{1}\left(x_{i}^{w}\right)= \begin{cases}w(2 n+3 m+2 t-1)-2 n-3 m-2 t+2 i & : 1 \leq i \leq \frac{n+1}{2} \quad ; 1 \leq w \leq s ; n \text { ganjil } \\ w(2 n+3 m+2 t-1)-2 n-3 m-2 t+2 i+1 & : \frac{n+3}{2} \leq i \leq n \quad ; 1 \leq w \leq s ; n \text { ganjil }\end{cases}
$$

$$
g\left(y_{j}^{w}\right)=w(2 n+3 m+2 t-1)-m+2 t-2 j+2 \quad: 1 \leq j \leq m \quad ; 1 \leq w \leq s
$$

$$
h\left(z_{k}^{w}\right)=w(2 n+3 m+2 t-1)-3 m-2 t+2 k+1 \quad: 1 \leq k \leq t \quad ; 1 \leq w \leq s
$$

$$
f_{2}\left(x_{i}^{w}\right)=\left\{\begin{array}{ccc}
w(2 n+3 m+2 t-1)-3 m-2 t-4 i & : 1 \leq i \leq \frac{n-2}{2} & ; 1 \leq w \leq s ; n \text { genap } \\
w(2 n+3 m+2 t-1)-2 n-3 m-2 t+2 & : i=\frac{n}{2} & ; 1 \leq w \leq s ; n \text { genap } \\
w(2 n+3 m+2 t-1)-4 n-3 m-2 t+4 i+3 & : \frac{n+2}{2} \leq i \leq n-2 ; 1 \leq w \leq s ; n \text { genap } \\
w(2 n+3 m+2 t-1)-3 m-2 t-2 & : i=n-1 & ; 1 \leq w \leq s ; n \text { genap } \\
w(2 n+3 m+2 t-1)-3 m-2 t+1 & : i=n & ; 1 \leq w \leq s ; n \text { genap }
\end{array}\right.
$$

Selanjutnya dari pelabelan titik tersebut, dengan definisi yang ada diperoleh pelabelan sisi sebagai berikut :

$$
\begin{aligned}
& f_{1}^{*}\left(e_{x, i}^{w}\right)=\left\{\begin{array}{cl}
w(2 n+3 m+2 t-1)-n-3 m-2 t+2 & : i=1 \\
w(2 n+3 m+2 t-1)-2 n-3 m-2 t+2 i-1 & : 2 \leq i \leq \frac{n+1}{2} ; 1 \leq w \leq s ; n \text { ganjil } \\
w(2 n+3 m+2 t-1)-2 n-3 m-2 t+2 i & : \frac{n+3}{2} \leq i \leq n ; 1 \leq w \leq s ; n \text { ganjil }
\end{array}\right. \\
& g^{*}\left(e_{y, j}^{w}\right)=\left\{\begin{array}{cc}
w(2 n+3 m+2 t-1)-m+2 t-2 j+1 & : 1 \leq j \leq m-1 ; 1 \leq w \leq s \\
w(2 n+3 m+2 t-1)-m-j+1 & : m \leq j \leq 2 m-1 ; 1 \leq w \leq s
\end{array}\right.
\end{aligned}
$$




$$
\begin{aligned}
& h^{*}\left(e_{z, k}^{w}\right)=\left\{\begin{array}{clr}
w(2 n+3 m+2 t-1)-3 m-2 t+2 & : k=1 & ; 1 \leq w \leq s \\
w(2 n+3 m+2 t-1)-3 m-2 t+2 k & : 2 \leq k \leq t & ; 1 \leq w \leq s
\end{array}\right. \\
& f_{2}^{*}\left(e_{x, i}^{w}\right)=\left\{\begin{array}{cll}
w(2 n+3 m+2 t-1)-3 m-2 t-1 & : i=1 & ; 1 \leq w \leq s ; \text { n genap } \\
w(2 n+3 m+2 t-1)-3 m-2 t-4 i+2 & : 2 \leq i \leq \frac{n-2}{2} & ; 1 \leq w \leq s ; n \text { genap } \\
w(2 n+3 m+2 t-1)-2 n-3 m-2 t+3 & : \frac{n}{2} \leq i \leq n-1 ; 1 \leq w \leq s ; n \text { genap } \\
w(2 n+3 m+2 t-1)-4 n-3 m-2 t+4 i+1 & : i=n & ; 1 \leq w \leq s ; \text { n genap }
\end{array}\right.
\end{aligned}
$$

Dengan demikian graf $s G C R_{m, n, t}$ adalah Super Mean. Contoh :
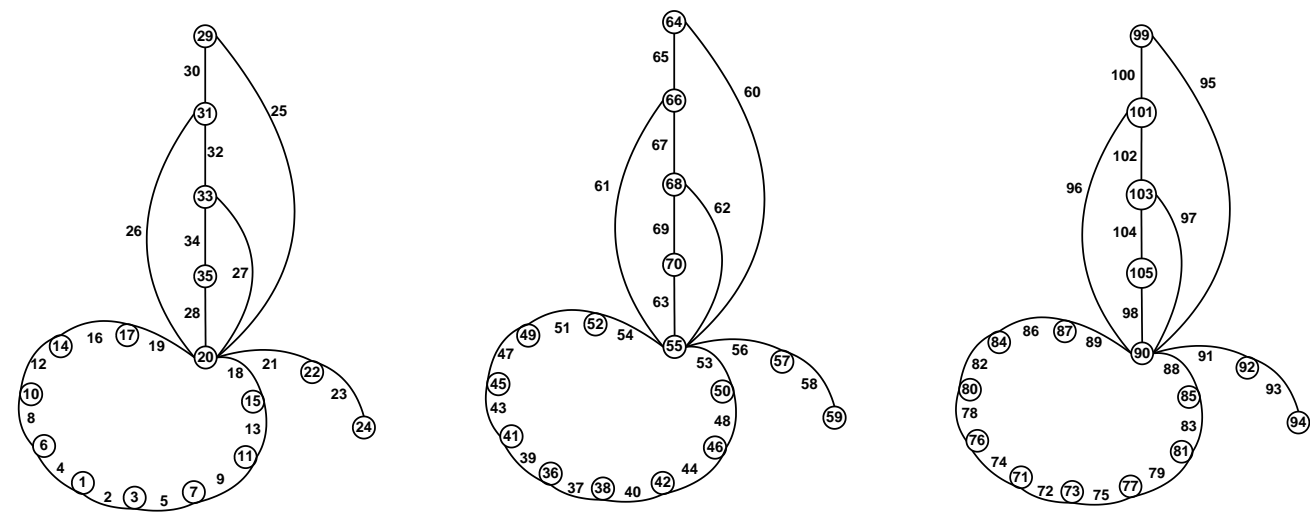

Gambar 2 : Graf Tunas Kelapa Gabungan $3 G C R_{10,4,2}$

3.1.2. Penotasian Titik dan Sisi Graf $G C R_{n, m, a, 2}$

Teorema 2 : $G C R_{n, m, a, 2}$ untuk $n \geq 7$ ganjil $m=n-3$, dan $a=2$ adalah Super Mean.

Bukti :

Berdasarkan penotasian titik dan sisi graf $G C R_{n, m, a, 2}$ untuk $a=2$ bentuk fungsi injektif $f\left(V\left(G C R_{n, m, 2,2}\right)\right) \cup\left\{f^{*}(e): e \in E\left(G C R_{n, m, 2,2}\right)\right\} \rightarrow\{3 n+2 m+4\}$ adalah sebagai berikut:

$\begin{array}{lc}f\left(x_{i}\right)=\left\{\begin{array}{lc}n+2 i+1 & : 1 \leq i \leq \frac{n-3}{2} \\ 2 n & : i=\frac{n-1}{2} \\ 2 i-n & : \frac{n+1}{2} \leq i \leq n\end{array}\right. \\ g\left(y_{i}\right)=3 n+2 m-2 j+6 & : 1 \leq j \leq m\end{array}$ 
$h\left(z_{k}^{\prime}\right)=\left\{\begin{array}{lll}3 n-2 k+4 & : \quad l=1 ; & 1 \leq k \leq 2 \\ 3 n+2 k+1 & : \quad l=2 ; & 1 \leq k \leq 2\end{array}\right.$

Dari pelabelan titik di atas diperoleh pelabelan sisi dengan fungsi :

$f^{*}\left(e_{x, i}\right)=\left\{\begin{array}{cl}n+2 & : i=1 \\ n+2 i & : i \leq i \leq \frac{n-3}{2} \\ 2 n-1 & : i=\frac{n-1}{2} \\ n+1 & : \frac{n+3}{2} \leq i \leq n\end{array}\right.$

$g^{*}\left(e_{y, j}\right)= \begin{cases}3 n+2 m-2 j+5 & : 1 \leq j \leq m-1 \\ 2 n+2 m-j+2 & : m \leq j \leq 2 m-1\end{cases}$

$h^{*}\left(e_{z, k}^{l}\right)= \begin{cases}2 n+1 & : \quad l=1 ; k=1 \\ 2 n+m+4 & : \quad l=1 ; k=2 \\ 2 n+2 & : \quad l=2 ; k=1 \\ 3 n+4 & : \quad l=2 ; k=2\end{cases}$

Dengan demikian graf $G C R_{n, m, 2,2}$ untuk $n \geq 7$, dan $m=n-3$ adalah Super Mean. Contoh :

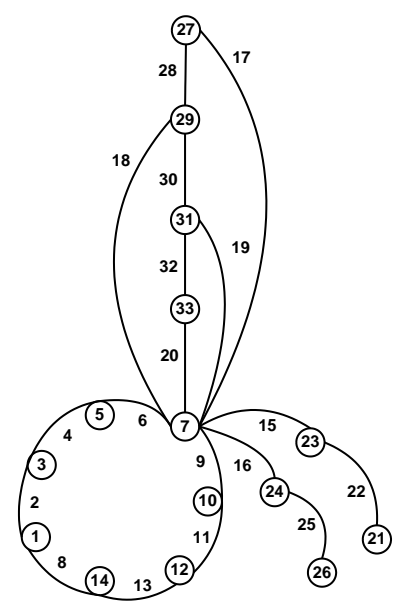

Gambar 3 : Pelabelan Super Mean Graf $G C R_{7,4,2,2}$ 
Teorema 3 : $G C R_{n, m, a, 2}$ untuk $n \geq 9$ ganjil, $m=n-6$, dan $a=3$ adalah Super Mean

\section{Bukti :}

Berdasarkan penotasian titik dan sisi graf $G C R_{n, m, a, 2}$ untuk $a=3$, bentuk fungsi injektif $f\left(V\left(G C R_{n, m, 3,2}\right)\right) \cup\left\{f^{*}(e): e \in E\left(G C R_{n, m, 3,2}\right)\right\} \rightarrow\{3 n+2 m+5\}$ adalah sebagai berikut:

$$
f\left(x_{i}\right)= \begin{cases}n+2 i+1 & : 1 \leq i \leq \frac{n-3}{2} \\ 2 n+1 & : i=\frac{n-1}{2} \\ 2 i-n & : \frac{n+1}{2} \leq i \leq n\end{cases}
$$

$g\left(y_{j}\right)=3 n+2 m-2 j+7 \quad: 1 \leq j \leq m$

$h\left(z_{k}^{l}\right)=\left\{\begin{array}{cll}3 n+2 k-4 & : 1=1 ; 1 \leq k \leq 2 \\ 3 n+3 l-2 k-1 & : 2 \leq l \leq 3 ; \quad 1 \leq k \leq 2\end{array}\right.$

Dari pelabelan titik di atas diperoleh pelabelan sisi dengan fungsi :

$$
\begin{gathered}
f^{*}\left(e_{x, i}\right)=\left\{\begin{array}{lc}
n+2 i & : i=1 \\
n+2 i & : i=\frac{n-1}{2} \\
\frac{3 n+2 i+1}{2} & : i=\frac{n+1}{2} \\
\frac{n+2 i+1}{2} & : \frac{n+3}{2} \leq i \leq n
\end{array}\right. \\
g^{*}\left(e_{y, j}\right)= \begin{cases}3 n+2 m-2 j+6 & : m \leq j \leq 2 m-1 \\
2 n+2 m-j+3 & : k=1 ; i=1\end{cases}
\end{gathered}
$$$$
h^{*}\left(e_{z, k}^{l}\right)= \begin{cases}2 n-1 & : k=1 ; l=1 \\ 3 n-1 & : k=2 ; \quad l=1 \\ 2 n+2 & : k=1 ; l=2 \\ 2 n+3 & : k=1 ; l=3 \\ 3 n+3 l-4 & k=2 \leq 1 \leq 3\end{cases}
$$

Dengan demikian graf $G C R_{n, m, a, 2}$ untuk $n \geq 9$ ganjil, $m=n-6$, dan $a=3$ adalah Super Mean. Contoh : 


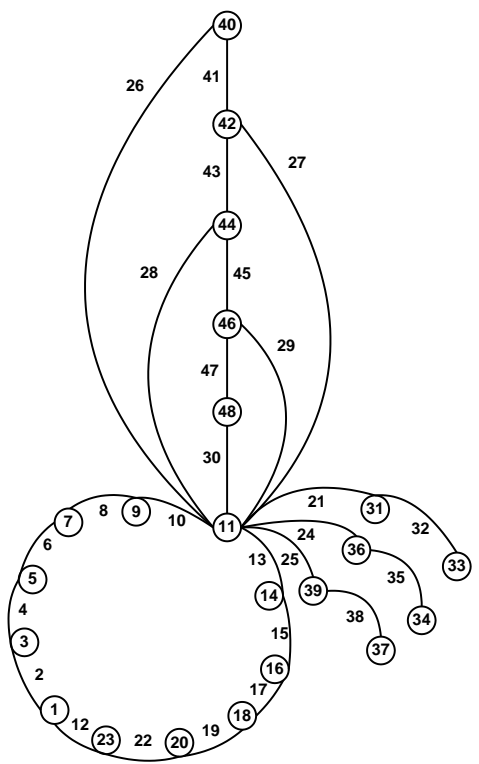

Gambar 4 : Pelabelan Super Mean Graf $G C R_{11,5,3,2}$

Teorema $4: G C R_{n, m, a, 2}$ untuk $n \geq 9$ ganjil, $m=n-7$, dan $a=4$ adalah Super Mean

\section{Bukti :}

Berdasarkan penotasian titik dan sisi graf $G C R_{n, m, a, 2}$ untuk $a=4$ pada Gambar 4.4 bentuk fungsi injektif $f\left(V\left(G C R_{n, m, 4,2}\right)\right) \cup\left\{f^{*}(e): e \in E\left(G C R_{n, m, 4,2}\right)\right\} \rightarrow$ $\{1,2,3, \ldots, 3 n+2 m+8\}$ adalah sebagai berikut:

$$
\begin{aligned}
& f\left(x_{i}\right)=\left\{\begin{array}{cc}
n+2 i+1 & : 1 \leq i \leq \frac{n-3}{2} \\
2 n+1 & : i=\frac{n-1}{2} \\
2 i-n & : \frac{n+1}{2} \leq i \leq n
\end{array}\right. \\
& g\left(y_{j}\right)=3 n+2 m-2 j+10 \quad: 1 \leq j \leq m \\
& \left(z_{k}^{l}\right)=\left\{\begin{array}{ccc}
3 n+2 k-4 & : \quad l=1 ; & 1 \leq k \leq 2 \\
3 n+3 l-2 k-1 & : \quad 2 \leq l \leq 3 ; & 1 \leq k \leq 2 \\
3 n+2 k+5 & : \quad l=4 ; & 1 \leq k \leq 2
\end{array}\right.
\end{aligned}
$$

Dari pelabelan titik di atas diperoleh pelabelan sisi dengan fungsi : 


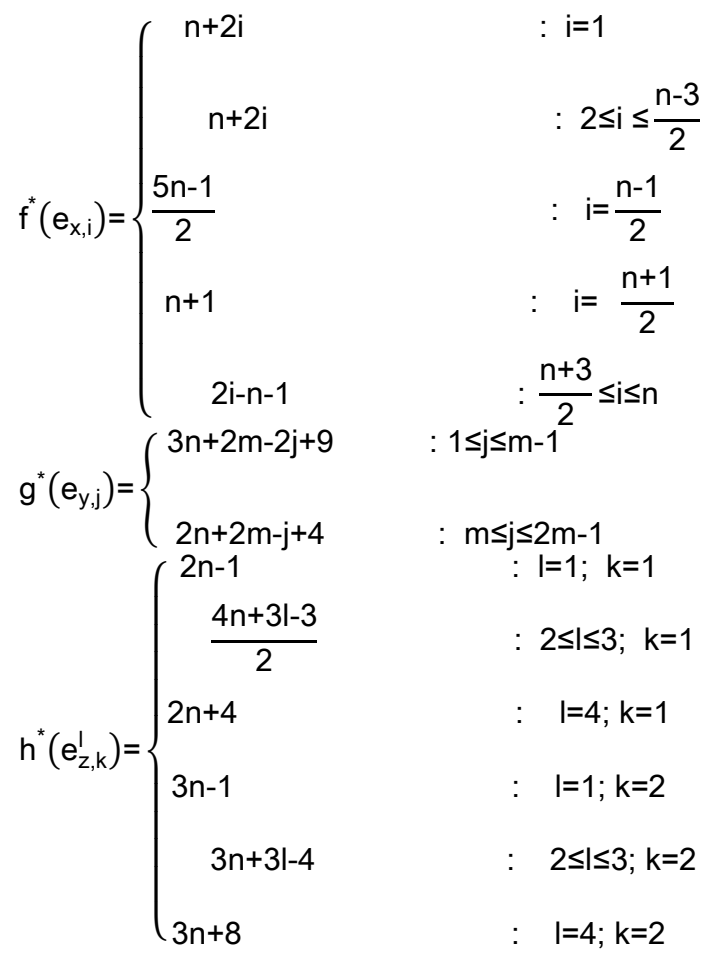

Contoh :

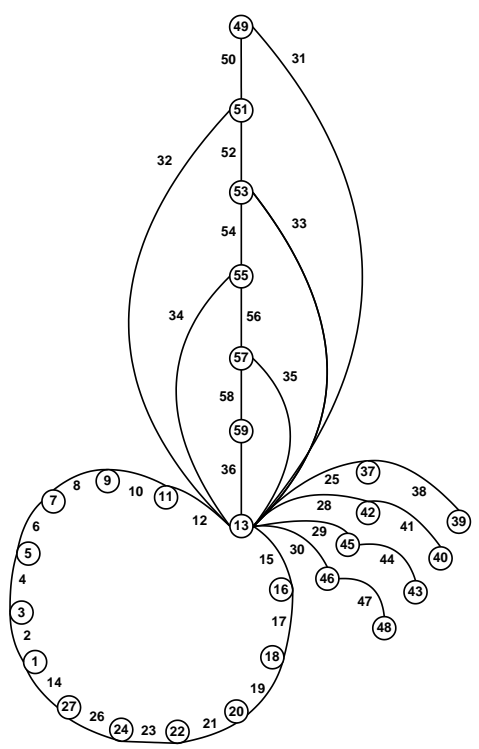

Gambar 5 : Pelabelan Super Mean Graf $G C R_{13,6,4,2}$ 
3.1.3. Penotasian Titik dan Sisi Graf $G C R_{n, m, a, 1} \cup P_{u}$

Teorema 5 : Graf $G C R_{n, m, a, 1} \cup P_{u}$ untuk $n \geq 9$ ganjil, $m=6, a=n-6$ dan $u=\frac{n-5}{2}$ adalah Super Mean.

\section{Bukti :}

Berdasarkan penotasian titik dan sisi graf $G C R_{n, m, a t} \cup P_{u}$ bentuk fungsi injektif $f\left(V\left(G C R_{n, m, t}\right)\right) \cup\left\{f^{*}(e): e \in E\left(G C R_{n, m, t}\right)\right\} \rightarrow\{1,2,3, \ldots, 3 n+2 a+11\} \quad$ adalah sebagai berikut:

$f\left(x_{i}\right)=\left\{\begin{array}{cc}n+2 i+1 & : 1 \leq i \leq \frac{n-1}{2} \\ 2 i-n & : \frac{n+1}{2} \leq i \leq n\end{array}\right.$

$g\left(y_{j}\right)=3 n+2 a-2 j+13 \quad: 1 \leq j \leq m$

$h\left(z_{k}^{\prime}\right)=3 n+2 l-1 \quad: 1 \leq 1 \leq a$

$\mathrm{q}_{1}\left(\mathrm{r}_{\mathrm{e}}\right)=3 \mathrm{n}+4 \mathrm{e}-2 \quad: 1 \leq \mathrm{e} \leq \mathrm{u}$

Dari pelabelan titik di atas diperoleh pelabelan sisi dengan fungsi :
$f^{*}\left(e_{x, i}\right)=\left\{\begin{array}{rr} & \\ 2 i+2 i & \\ & 2 i-n-1\end{array}\right.$
$g^{*}\left(e_{y, j}\right)=\left\{\begin{array}{lc}3 n+2 a-2 j+12 & : 1 \leq j \leq m-1 \\ 2 n+a+m-j+6 & : m \leq j \leq 2 m-1\end{array}\right.$
$h^{*}\left(e_{z, k}^{l}\right)=2 n+1$
$: 1 \leq 1 \leq a$
$q^{*}\left(e_{r, e}\right)=3 n+4 e$
: $1 \leq \mathrm{e} \leq \mathrm{u}-1$

Dengan demikian Graf $G C R_{n, m, a, 1} \cup P_{u}$ untuk $n \geq 9$ ganjil, $m=6, a=n-6$ dan $u=\frac{n-5}{2}$ adalah Super Mean. Contoh :

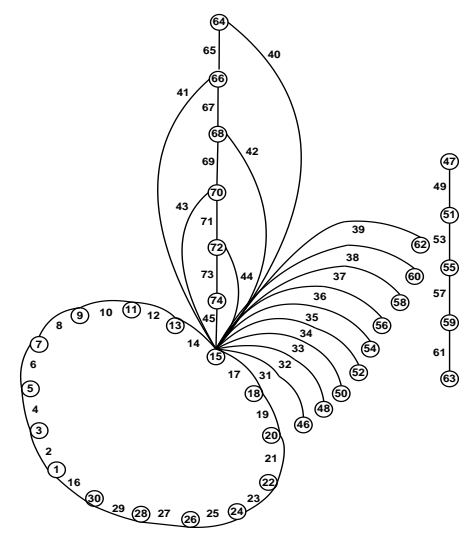




$$
\text { Gambar } 6 \text { : Pelabelan Super Mean Graf } G C R_{15,6,9,1} \cup P_{5}
$$

\section{KESIMPULAN}

Berdasarkan hasil yang didapatkan dalam penelitian ini, maka dapat dibuat beberapa kesimpulan sebagai berikut:

1. Graf $s G C R_{n, m, t}$ untuk $n \geq 5, m \geq 2, t=\left\lfloor\frac{m}{2}\right\rfloor$ dan $s \geq 1$ adalah Super Mean

2. $G C R_{n, m, a, 2}$ untuk $n \geq 7$ ganjil $m=n-3$, dan $a=2$ adalah Super Mean

3. $G C R_{n, m, a, 2}$ untuk $n \geq 9$ ganjil, $m=n-6$, dan $a=3$ adalah Super Mean

4. $G C R_{n, m, a, 2}$ untuk $n \geq 9$ ganjil, $m=n-7$, dan $a=4$ adalah Super Mean

5. $G C R_{n, m, a, 1} \cup P_{u}$ untuk $n \geq 9$ ganjil, $m=6, a=n-6$ dan $u=\frac{n-5}{2}$ adalah Super Mean

\section{DAFTAR PUSTAKA}

[1]. Lestari, I. L., 2013. Pelabelan Total Super (a,d)-Sisi Anti Magic Graf Tunas Kelapa. Skripsi tidak diterbitkan. Jember : Program Studi Pendidikan Matematika Fakultas Keguruan dan IImu Pendidikan, Universitas Jember.

[2]. Ramya D., and Jeyanthi P.,, 2012, Super Mean Labeling of Some Classes Graphs, International J. Math. Combin, Vol.1, 83-91.

[3]. Wallis, W.D., Baskoro, Edy T., Miller., and Slamin., 2000, Edge-Magic Total Labeling, Australian Journal of Combinatorics, Vol. 22, 177-190. 